\title{
Gradual reduction of testosterone using a gonadotropin-releasing hormone vaccination delays castration resistance in a prostate cancer model
}

\author{
JESÚS A. JUNCO BARRANCO ${ }^{1}$, ROBERT P. MILLAR ${ }^{2,3}$, FRANKLIN FUENTES ${ }^{1}$, EDDY BOVER ${ }^{1}$, \\ EULOGIO PIMENTEL ${ }^{4}$, ROBERTO BASULTO ${ }^{1}$, LESVIA CALZADA ${ }^{1}$, ROLANDO MORÁN ${ }^{1}$, AYNI RODRÍGUEZ ${ }^{5}$, \\ HILDA GARAY ${ }^{4}$, OSVALDO REYES ${ }^{4}$, MARIA D. CASTRO $^{1}$, RICARDO BRINGAS $^{4}$, NIURKA ARTEAGA ${ }^{1}$, \\ HENIO TOUDURI ${ }^{5}$, MAURICIO RABASSA ${ }^{1}$, YAIRIS FERNÁNDEZ ${ }^{5}$, ANDRÉS SERRADELO ${ }^{1}$, \\ EDUARDO HERNÁNDEZ ${ }^{1}$ and GERARDO E. GUILLÉN ${ }^{4}$
}

\footnotetext{
${ }^{1}$ Department of Vaccines, Center for Genetic Engineering and Biotechnology, Camagüey, Camagüey 70100, Cuba;

${ }^{2}$ Mammal Research Institute and Centre for Neuroendocrinology, University of Pretoria, Pretoria, Gauteng 0028;

${ }^{3}$ MRC Receptor Biology Unit, Institute for Infectious Diseases and Molecular Medicine, University of Cape Town, Cape Town, Western Cape 7925, South Africa; ${ }^{4}$ Center for Genetic Engineering and Biotechnology,

Havana, Havana 10600; ${ }^{5}$ Medical University of Camagüey, Camagüey, Camagüey 70100, Cuba
}

Received February 23, 2015; Accepted December 16, 2015

DOI: $10.3892 / \mathrm{ol} .2016 .4679$

\begin{abstract}
In a previous study aimed to design a novel prostate cancer vaccine, the authors of the present study demonstrated the advantage of combining the adjuvants Montanide ISA 51 with very small size proteoliposomes (VSSP) to promote a significant humoral immune response to gonadotropin-releasing hormone $(\mathrm{GnRH})$ in healthy animals. The present study compared the efficacy of this vaccine formulation versus the standard treatment currently available in terms of preventing the development of tumors in $\mathrm{DD} / \mathrm{S}$ mice injected with Shionogi carcinoma (SC) 115 cells. The results demonstrated that 5 non-vaccinated control mice exhibited a fast tumor growth, and succumbed to the disease within 19-31 days. Mice immunized with the GnRH/Montanide ISA 51/VSSP vaccine exhibited a moderate decline in testosterone levels that was associated with a decrease in anti-GnRH antibody titers, which lead to a sustained tumor growth inhibition. In total, 2 mice in the immunized group exhibited complete remission of the tumor for the duration of the present study. In addition, castrated mice, which were used as a control for standard hormonal therapy, exhibited an accelerated decrease in tumor size. However, tumor relapse was observed between days 50 and 54, and between days 65 and 85, following the injection of
\end{abstract}

Correspondence to: Dr Jesús A. Junco Barranco, Department of Vaccines, Center for Genetic Engineering and Biotechnology, Finlay and North Ring Avenue s/n, Camagüey, Camagüey 70100, Cuba E-mail: jesus.junco@cigb.edu.cu

Key words: prostate cancer vaccine, gonadotropin-releasing hormone vaccination, adjuvant combination, hormone ablation, castration-resistant prostate cancer
SC 155 cells. Therefore, these mice were sacrificed at day 90 . The present study concludes that the slow and moderate reduction of testosterone levels observed using the GnRH-based vaccine may delay the appearance of castration resistance in a Shionogi prostate cancer model. These findings suggest that this vaccine may be used to delay castration resistance in patients with prostate cancer.

\section{Introduction}

Prostate cancer is the most commonly diagnosed malignancy in elderly men, and the second leading cause of cancer-associated mortality in the Western world $(1,2)$.

Standard treatments for prostate cancer include surgery, radiation therapy and androgen deprivation therapy (ADT), which are usually successful in controlling organ-confined disease. However, in $30 \%$ of patients the disease recurs and becomes androgen deprivation-resistant $(3,4)$.

Since the 1990's, gonadotropin-releasing hormone (GnRH) analogues have been the most successful drugs for achieving ADT (5). Androgen is required for the function and maintenance of prostate cancer cells, and androgen deprivation results in an inhibition of growth and decrease in volume of prostate cancer tumors (6-8). However, at advanced stages of the disease, almost all tumors develop into castration-resistant prostate cancer (CRPC), the management of which is a great challenge $(6,8,9)$. Consequently, there is a clear requirement for improved treatments to prevent the development of CRPC, and for novel therapies to treat patients with prostate cancer.

GnRH-based vaccines represent a promising anti-hormonal treatment alternative for the treatment of prostate cancer, since they reduce serum testosterone levels and avoid the flare response observed when using GnRH analogues (6,10-12). In addition, following the completion of the immunization 
procedure, the patient does not require the administration of any other medication for a prolonged period, which results in low costs $(6,12)$. Therefore, these types of vaccines may be highly competitive in the market $(10,11,13)$. However, the development of therapeutic vaccines for cancer therapy presents disadvantages, due to the low immunogenicity of antigens, tolerance induction and heterogeneity of results among individuals $(6,14,15)$.

The use of adjuvant combinations such as aluminum salts (16) and squalene oil (17), alone or in combination with saponins, has been recently employed in the development of preventive vaccines, including the vaccine for human papilloma virus (18-20). However, adjuvants have not been used for the development of therapeutic vaccines for advanced cancer thus far.

The combination of Montanide ISA 51, a type 1 adjuvant, and very small size proteoliposomes (VSSP), a type 2 adjuvant that is derived from the fermentation of Neisseria meningitides, has been recently used by the authors of the present study for the generation of anti-GnRH antibodies in healthy animals with excellent results (12).

The present study demonstrates the humoral immune response against the autologous $\mathrm{GnRH}$ hormone, and its effect on the inhibition of testosterone levels and tumor development in DD/S male mice that were injected with hormone-sensitive Shionogi carcinoma (SC) 115 cells. The SC 115 mice received 6 immunizations of the GnRH mimetic peptide (GnRHm1-TT)/Montanide ISA 51/VSSP vaccine candidate. The SC 115 mouse model is a transplantable androgen-dependent neoplasia similar to human tumors, which may develop into CRPC in $>90 \%$ of cases, despite the use of the maximal androgen blockade $(21,22)$. The present study also measured the reactivity of specific immune response to a $40 \mathrm{kDa}$ antigen, which had been previously reported to be induced by castration, using western blot and interferon (IFN)- $\gamma$ enzyme-linked immunospot (ELISPOT) assays $(9,22)$.

\section{Materials and methods}

Generation of the synthetic GnRHml-TT. GnRHm1-TT was synthesized using conventional solid phase synthesis (6), at the Center for Genetic Engineering and Biotechnology of Havana (Havana, Cuba). L-glycine at position 6 of the natural GnRH (sequence, QHWSYGLRPG) was substituted for L-proline (sequence, QHWSYPLRPG), and the epitope QYIKANSKFIGITEL of tetanus toxoid was added to the carboxyl terminus (6).

For the vaccine emulsion preparation, $750 \mu \mathrm{g}$ lyophilized GnRHm1-TT was resuspended in distilled water and mixed with $120 \mu \mathrm{g}$ VSSP (obtained from the Center for Molecular Immunology, Havana, Cuba) to a final volume of $100 \mu 1$. The same quantity of Montanide ISA 51 (Seppic, Paris La Défense, France) was added in a 1:1 ratio. The vaccine formulation was centrifuged (SCT 15B Himac centrifuge; Hitachi Medical Corporation, Tokyo, Japan) at 3,500 x g for $30 \mathrm{~min}$. The final emulsion was administered to the mice by subcutaneous injection at four points of the dorsal region. For the placebo preparation, an identical procedure was performed with the exception of the peptide, which was not included in the mixture.
Animal models and experimental groups. In total, 15 male DD/S mice were maintained at the Animal Care Unit of the University of Victoria (Victoria, Canada). All protocols followed the guidelines of the Canadian Council for Animal Care (Ottawa, Canada; approval no., NOVO7BHN) and were approved by the Animal Care Committee at the University of Victoria. The animals were housed two per cage, under humidity and temperature controlled conditions and a light/dark cycle of $12 \mathrm{~h}$ intervals. The DD/S mice, which were 8-10 weeks old, were randomized into 3 groups of 5 mice each, as follows: Group 1 received 5 subcutaneous injections of the vaccine candidate GnRHm1-TT/Montanide ISA 51/VSSP (12); group 2 received the placebo, which was administered using the same immunization schedule as group 1; and group 3 was surgically castrated once the tumor reached $8-10 \mathrm{~mm}$ in diameter.

$\mathrm{DD} / \mathrm{S}$ mice were injected with SC 115 cells, which were cultured according to the protocol described by Nesslinger et al (22), on day 40 subsequent to the beginning of the experiment, when the immunized and placebo groups had received 3 immunizations. For the tumor implantation, $5 \times 10^{6}$ cells in phosphate-buffered saline (PBS; EMD Millipore, Billerica, MA, USA) were injected subcutaneously into the flank of the mice, according to the protocol by Nesslinger et al (22). The tumor was measured daily using a digital calliper. The tumor volume was calculated using the following formula: Tumor volume $=4 / 3 \pi \times$ tumor radius ${ }^{3}$. When the tumor reached a volume $\geq 1.5 \mathrm{~cm}^{3}$, the mice were sacrificed using anaesthesia. Tumors that were palpable were considered to be recurring tumors.

Blood extraction and serum collection. Blood was extracted from the mice $(\sim 100 \mu \mathrm{l})$ by puncturing the radial venous with a 26G needle (Terumo Medical Corporation, Tokyo, Japan), and using heparinized micro-hematocrit tubes (Drummond Scientific Company, Broomall, PA, USA). The blood was centrifuged at 3,200 x $\mathrm{g}$ for $15 \mathrm{~min}$ to obtain the serum, which was stored at $-20^{\circ} \mathrm{C}$ until further use. Splenocytes from the mice were isolated according to the protocol described by Nesslinger et al (22).

Anti-GnRH antibody determination. An enzyme-linked immunosorbent assay (ELISA) was performed using high-binding Nunc ${ }^{\mathrm{TM}}$ 96-well polystyrene plates (Thermo Fisher Scientific, Inc., Waltham, MA, USA), which were coated overnight with $10 \mu \mathrm{g} / \mathrm{ml}$ natural $\mathrm{GnRH}$ peptide (Center for Genetic Engineering and Biotechnology of Havana) at $4^{\circ} \mathrm{C}$ in carbonate/bicarbonate buffer $(0.1 \mathrm{M}$; pH 9.6; Merck, Kenilworth, NJ, USA). The serum samples were diluted to $1: 50$ in PBS (0.1 M; pH 7.4) for seroconversion, and between 1:1,000 and 1:20,000 for anti-GnRH titration in PBS (0.1 M; pH 7.4) containing $2 \%$ bovine serum albumin (BSA; Sigma-Aldrich, St Louis, MO, USA). The plates were incubated for $3 \mathrm{~h}$ at $37^{\circ} \mathrm{C}$. Subsequently, polyclonal rabbit anti-mouse immunoglobulin (Ig) $\mathrm{G}$ antibody conjugated to peroxidase (cat. no. A9044; Sigma-Aldrich), which was diluted to $1: 10,000$ in PBS (0.1 M; pH 7.4) and $2 \%$ BSA, was added to the plates. The antigen-antibody reaction was detected by addition of o-phenylenediamine (EMD Millipore) and a $\mathrm{H}_{2} \mathrm{O}_{2}$ substrate (EMD Millipore), 
which was dissolved in disodium phosphate (0.02 M, pH 5.0; EMD Millipore). The colorimetric reaction was read at $492 \mathrm{~nm}$ using a plate reader (Multiskan ${ }^{\mathrm{TM}}$ GO Microplate Spectrophotometer; Thermo Fisher Scientific, Inc.). Samples were considered to be positive when the absorbance values were $>0.197$, which was the cut-off.

Testosterone determination. Testosterone levels were determined using the Testosterone radioimmunoassay kit (TESTO-CT2 assay; Cisbio Bioassays, Codolet, France). The sensitivity of the method, defined as the detectable concentration equivalent to twice the standard deviation of the zero-binding value, was $\sim 0.1 \mathrm{nmol} / 1$. The specificity of the kit for testosterone was $>99 \%$. To carry out the determination, $25 \mu 1$ serum from each sample was plated directly in the pre-coated tubes provided in the kit. Duplicates from all the samples were incubated for $1 \mathrm{~h}$ at $37^{\circ} \mathrm{C}$. The tubes were washed 3 times with distilled water at room temperature, and read in a gamma counter (HITACHI-ALOKA AccuFLEX LSC-8000 Scintillation Counter; Hitachi Medical Corporation). The results were recorded as nmol/l.

Western blot analysis to detect anti-Shionogi proteins. Western blot analysis was performed as previously described (23). Briefly, proteins $(400 \mu \mathrm{g})$ from SC 115 tumor cells (obtained from the Nelson Lab, University of Victoria, Victoria, Canada) were separated using $12 \%$ standard polyacrylamide gel electrophoresis (Bio-Rad Laboratories, Inc., Hercules, CA, USA), and transferred to nitrocellulose membranes (Thomas Scientific, Swedesboro, NJ, USA. A molecular weight marker 20-80 KD (Sigma-Aldrich) was used. The sera of the mice from the various groups were diluted in dilution buffer (dilution, 1:500), which contained PBS (50 mM), 5\% dry milk powder (Sigma-Aldrich), 0.1\% Tween 20 (EMD Millipore), 50 mmol/1 Tris (EMD Millipore) and $150 \mathrm{mmol} / \mathrm{l}$ sodium chloride (EMD Millipore), and incubated for $1 \mathrm{~h}$ at room temperature with the nitrocellulose membrane, using the Mini Protean $\mathrm{II}^{\circledR}$ MultiScreen Apparatus (Bio-Rad Laboratories, Inc., Hercules, CA, USA). The nitrocellulose membrane was washed, incubated for $1 \mathrm{~h}$ at room temperature with polyclonal horseradish peroxidase-conjugated goat anti-mouse IgG secondary antibody (H+L; cat. no. 115-035-003; dilution, 1:10,000; Jackson ImmunoResearch Laboratories, Inc., West Grove, PA, USA), and visualized using enhanced chemiluminescence.

IFN- $\gamma$ ELISPOT assay. MultiScreen-IP Filter Plates (96 wells; $0.45 \mu \mathrm{m}$ pore size; EMD Millipore, Billerica, MA, USA) were pre-wet with $70 \%$ ethanol, followed by three washes with sterile PBS (Gibco ${ }^{\circledR}$; Thermo Fisher Scientific, Inc.). The plates were incubated overnight at $4^{\circ} \mathrm{C}$ with $50 \mu \mathrm{l} /$ well monoclonal rat anti-mouse IFN- $\gamma$ antibody $(10 \mu \mathrm{g} / \mathrm{ml}$; clone, AN18; catalog no., 3321-3-250; Mabtech, Inc., Cincinnati, $\mathrm{OH}$, USA). Following three washes with PBS, the plates were blocked with T-cell medium (Sigma-Aldrich; RPMI 1640 supplemented with $10 \%$ fetal bovine serum, $1 \mathrm{mM}$ sodium pyruvate, $2 \mathrm{mM}$ L-glutamine, $100 \mu \mathrm{g} / \mathrm{ml}$ penicillin/streptomycin and $25 \mu \mathrm{M} 2$-mercaptoethanol) for $2 \mathrm{~h}$ at $37^{\circ} \mathrm{C}$. In total, $3 \times 10^{5}$ splenocytes from the mice were added to each well. Polyadenylate-binding protein nuclear 1 (PABPN1; the Nelson Lab, University of Victoria) and concanavalin A
(Sigma-Aldrich) were added to the wells at a final concentration of 10 and $2 \mu \mathrm{g} / \mathrm{ml}$, respectively. T-cell medium was used as a negative control. The samples were run in triplicate. The plates were incubated for $24 \mathrm{~h}$ at $37^{\circ} \mathrm{C}$. Subsequent to washing six times with $50 \mathrm{mM}$ PBS containing $0.05 \%$ Tween 20 , $100 \mu \mathrm{l}$ biotinylated anti-mouse IFN- $\gamma$ was added to each well. The plates were incubated for $2 \mathrm{~h}$ at $37^{\circ} \mathrm{C}$, and then washed 12 times with PBS at room temperature containing $0.05 \%$ Tween 20. Avidin-biotin peroxidase complex (VECTASTAIN $^{\circledR}$ ABC kit; Vector Laboratories, Inc., Burlingame, CA, USA) was added to the plates for 5-10 $\mathrm{min}$. The development was stopped by rinsing the plates with water. Air-dried plates were read for enumeration of spots using an ELISPOT reader with KS ELISPOT 4.4 software (Carl Zeiss AG, Oberkochen, Germany).

Statistical analysis. For the statistical analysis to enable a parametric evaluation of the data, analysis of variance and Student's $t$ tests were used. The treatment groups were compared using the Student's $t$-test. For animal survival analysis Kaplan-Meier analysis was used in addition to the $\log$ rank test for statistical associations. A one-way analysis of variance was used for antibody and testosterone analysis. Prism GraphPad version 5.0 (GraphPad Software, Inc., La Jolla, CA, USA) was used to conduct statistical analysis. $\mathrm{P}<0.05$ was considered to indicate a statistically significant difference.

\section{Results}

Anti-GnRH antibodies, generated by the vaccine candidate GnRHm1-TT/Montanide ISA 51/VSSP, induce testosterone ablation in DD/S mice implanted with SC 115 tumors. The development of the vaccine formulation GnRHm1-TT/Montanide ISA 51/VSSP is a promising candidate to generate a productive response against $\mathrm{GnRH}$, which presents the advantage of combining a type 1 and a type 2 adjuvants $(12,24)$. To investigate how this vaccine candidate affects a tumoral model, the present study performed an experiment in DD/S mice implanted with the hormone-sensitive SC 115 tumor cell line, which has been extensively used to study the conversion from androgen-dependent to androgen-independent neoplasia $(7,21)$. Shionogi tumors are initially androgen-dependent, and are therefore grown in male mice $(8,9,21,22)$. Castration of the mice leads to apoptosis and tumor regression similar to that observed following androgen withdrawal in patients with prostate cancer $(8,21)$.

The effect of the GnRHm1-TT/Montanide ISA 51/VSSP vaccine on anti-GnRH antibodies, testosterone levels and tumor size in DD/S mice was evaluated in the present study. In all 5 mice, $100 \%$ of anti-GnRH seroconversion was achieved following the administration of 2 immunizations (Table I). The anti-GnRH immune response was investigated using an in-house titration indirect ELISA method $(6,12)$, which rendered titers between 1:1,000 and 1:4,000 (Table I). Similarly to the anti-GnRH immune response, the measurement of the testosterone levels by radioimmunoassay demonstrated a decrease in the testosterone concentration of the immunized animals, although the levels of testosterone in these animals were higher than those detected in the castrated mice (Table I). 
Table I. Effects of various treatments on the anti-GnRH seroconversion, testosterone levels, survival time and anti-PABPN1 antibody titers of DD/S mice injected with hormone-sensitive Shionogi carcinoma 115 tumor cells.

\begin{tabular}{lccccc}
\hline Animal no. & $\begin{array}{c}\text { Experimental } \\
\text { group }\end{array}$ & $\begin{array}{c}\text { Anti-GnRH, } \\
\text { titer }\end{array}$ & $\begin{array}{c}\text { Testosterone levels, } \\
\text { nmol/l }\end{array}$ & $\begin{array}{c}\text { Survival time, } \\
\text { days }\end{array}$ & $\begin{array}{c}\text { Anti-PABPN1, } \\
\text { western blot signal }\end{array}$ \\
\hline 1 & Placebo & 0 & $17.30 \pm 0.1^{\mathrm{c}}$ & $31^{\mathrm{c}}$ & $\mathrm{ND}$ \\
2 & Placebo & 0 & $16.30 \pm 0.1^{\mathrm{c}}$ & $19^{\mathrm{c}}$ & $\mathrm{ND}$ \\
3 & Placebo & 0 & $17.40 \pm 0.2^{\mathrm{c}}$ & $27^{\mathrm{c}}$ & - \\
5 & Placebo & 0 & $12.90 \pm 0.0^{\mathrm{c}}$ & $19^{\mathrm{c}}$ & +++ \\
8 & Placebo & 0 & $13.40 \pm 0.0^{\mathrm{c}}$ & $24^{\mathrm{c}}$ & + \\
11 & Immunized & $1: 1,000^{\mathrm{c}}$ & $1.97 \pm 0.1^{\mathrm{b}}$ & $30^{\mathrm{c}}$ & $\mathrm{ND}$ \\
12 & Immunized & $1: 4,000^{\mathrm{a}}$ & $1.63 \pm 0.1^{\mathrm{b}}$ & $100^{\mathrm{a}}$ & - \\
13 & Immunized & $1: 2,000^{\mathrm{b}}$ & $1.74 \pm 0.2^{\mathrm{b}}$ & $35^{\mathrm{c}}$ & - \\
14 & Immunized & $1: 4,000^{\mathrm{a}}$ & $0.89 \pm 0.0^{\mathrm{a}}$ & $100^{\mathrm{a}}$ & - \\
17 & Immunized & $1: 4,000^{\mathrm{a}}$ & $1.04 \pm 0.0^{\mathrm{a}}$ & $55^{\mathrm{b}}$ & +++ \\
26 & Castrated & 0 & $0.07 \pm 0.1^{\mathrm{a}}$ & $90^{\mathrm{a}}$ & ++ \\
27 & Castrated & 0 & $0.23 \pm 0.1^{\mathrm{a}}$ & $70^{\mathrm{b}}$ & - \\
28 & Castrated & 0 & $0.74 \pm 0.2^{\mathrm{a}}$ & $90^{\mathrm{a}}$ & - \\
29 & Castrated & 0 & $0.89 \pm 0.0^{\mathrm{a}}$ & $59^{\mathrm{b}}$ & +++ \\
30 & Castrated & 0 & $0.47 \pm 0.0^{\mathrm{a}}$ & $90^{\mathrm{a}}$ & $\mathrm{ND}$ \\
\hline
\end{tabular}

Differences in anti-GnRH antibody titers and testosterone levels measured at day 60 were calculated using one-way analysis of variance, while a log-rank test was performed to calculate the differences in survival time. Anti-PABPN1 antibodies were detected using western blot analysis. ${ }^{\mathrm{a}} \mathrm{P}<0.001,{ }^{\mathrm{b}} \mathrm{P}<0.01,{ }^{\mathrm{c}} \mathrm{P}<0.05$, according to the Mann-Whitney $\mathrm{U}$ test. Anti-PABPN1 antibodies were determined using a western blot analysis. GnRH, gonadotropin-releasing hormone; PABPN1, polyadenylate-binding protein nuclear 1; ND, not determined; +, presence of anti-PABPN1 antibodies; +++, marked presence of anti-PABPN1 antibodies; -, absence of anti-PABPN1 antibodies.

GnRHm1-TT/Montanide ISA 51/VSSP produces tumor growth inhibition in SC 115 mice. The present study investigated the association between testosterone ablation induced by anti-GnRH antibodies and tumor growth rate. The present study observed that in the immunized group, 3 out of 5 mice exhibited a significant decrease in tumor size $(\mathrm{P}<0.01)$ (Fig. 1A). The tumor volume reduced to $>200 \mathrm{~mm}^{3}$ at day 30 $(\mathrm{P}<0.05)$. These 3 slow-growing tumors exhibited a continuous decrease in size over time, and completely regressed at day 65 following the injection of SC 115 cells. There was no recurrence of the tumor by the end of the study at day 100. In total, 1 of the 5 immunized mice was sacrificed prior to the end of the present study (day 55), since the mouse mutilated the tumor implanted region. The remaining 2 mice in the immunized group exhibited an accelerated tumor growth, and had to be sacrificed on day 30 and 35, respectively (Fig. 1A).

In the castrated group, 4 out of 5 mice exhibited tumor regression 10 days following castration. The maximum reduction in tumor volume was observed at day $30\left(180 \mathrm{~mm}^{3}\right)$. In the remaining mice, $\sim 40$ days were required to achieve a similar reduction in tumor size (Fig. 1B). However, there was tumor regrowth in 2 mice at days 50 and 54 . Therefore, the mice were sacrificed at days 59 and 70, respectively (Table I). The remaining 3 mice experienced tumor regrowth from day 70 , and had to be sacrificed on day 90 (Fig. 1B). The entire placebo group had to be sacrificed by $\sim$ day 30 post-tumor cell injection, since all the mice exhibited an accelerated tumor growth (Fig. 1C).

The comparison in survival time between the placebo, surgically castrated and immunized mice was calculated using the log-rank test. The results demonstrated that the immunized and castrated animals survived for twice as long as the placebo group $(\mathrm{P}<0.01)$. The surgically castrated and vaccinated mice did not exhibit any significant differences in survival time $(\mathrm{P}>0.05)$. However, it is notable that in the vaccinated group there were 2 animals in which tumors did not recur for the duration of the present study (Fig. 2).

Anti-PABPN1 immune response in DD/S mice implanted with SC 115 tumor cells is not associated with improvements in tumor control or survival benefits. Previous studies have implicated anti-PABPN1 antibodies in the development of Shionogi carcinoma. Therefore, the present study investigated whether the development of hormone therapy-associated serological responses was associated with the extent of tumor regression. The present study compared the SC 115 tumor-implanted $\mathrm{DD} / \mathrm{S}$ mice that had received the standard castration treatment with the GnRHm1-TT/Montanide ISA 51/VSSP vaccine and the placebo groups. The results demonstrated that for the immunized group, 1 out of 4 mice (25\%) developed anti-PABPN1 antibodies (mouse no., 17; Fig. 3A), compared with 2 out of 4 mice (50\%) in the castrated group (mice no., 26 and 29; Fig. 3B and C, respectively), and 2 out of 3 mice (66\%) in the placebo group. (mice no., 5 and 8; Fig. 3A and D, respectively).

According to these results, there was no association between the presence of anti-PABPN1 antibodies and the tumor growth rate. However, a poor prognosis for tumor growth was observed in mice that developed an immune response against PABPN1 (Fig. 3A-D). Notably, in mice treated with the 

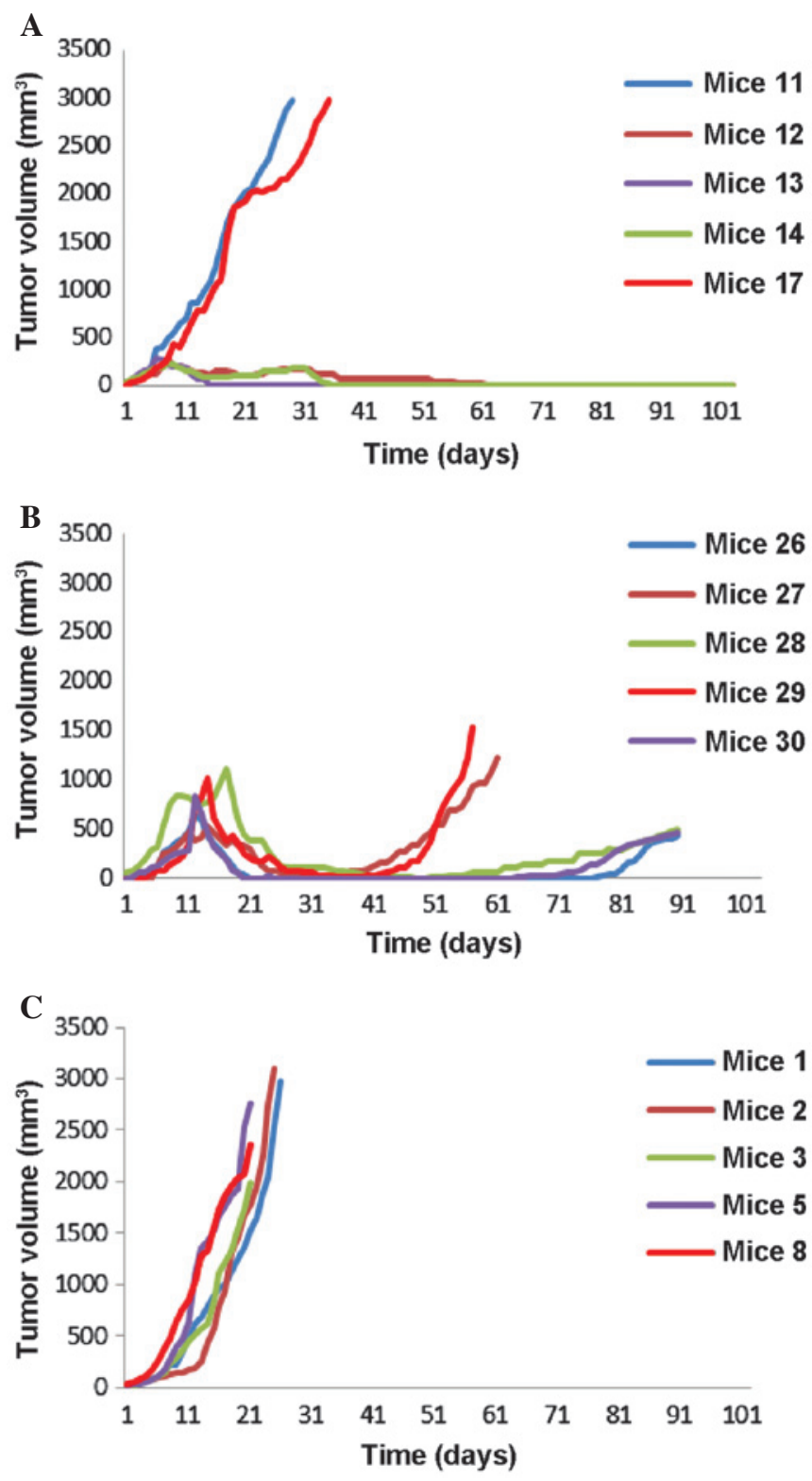

Figure 1. Tumor growth rate of the Shionogi carcinoma 115-implanted DD/S mice subjected to various treatments. (A) Mice that received vaccine candidate GnRHm1-TT/Montanide ISA 51/VSSP at days 0, 15, 30, 45 and $60(n=5)$. It was observed that 2 out of 5 mice exhibited fast-growing tumors in the first 30 days, and 2 out of 5 mice exhibited tumor growth inhibition until day 100 . (B) Castrated mice, used as control $(n=5)$. The mice were castrated 15 days following tumor implantation. Tumor growth was inhibited in all these mice, although tumor re-growth was observed on days 59-90. (C) Placebo mice, which were immunized with a mixture of Montanide ISA 51/VSSP $(n=5)$. All the mice exhibited a fast tumor growth, and had to be sacrificed prior to day 31 following tumor implantation. VSSP, very small size proteoliposomes; GnRHm1-TT, gonadotropin-releasing hormone mimetic peptide.

vaccine candidate GnRHm1-TT/Montanide ISA 51/VSSP, the mice that exhibited the most accelerated tumor growth were those that developed anti-PABPN1 antibodies, as measured by western blot analysis (mouse no., 17; Fig. 3B). Similarly, in the placebo group, which presented the highest tumor growth rate, the highest anti-PABPN1 immune response was detected (mouse no., 5 and 8; Fig. 3A and D, respectively). For the surgically castrated mice, a similar association between anti-PABPN1 immune response and survival time was observed (mouse no., 26 and 29; Fig. 3B and C, respectively).

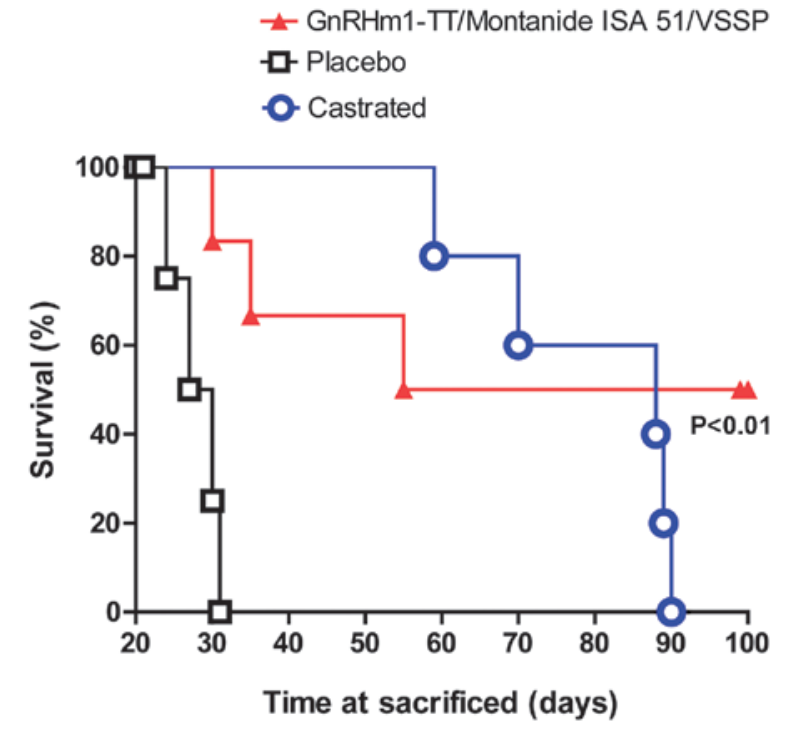

Figure 2. Kaplan-Meier representation of the survival time for various treatment groups. Mice in the vaccinated group were immunized 6 times with the vaccine candidate GnRHm1-TT/Montanide ISA 51/VSSP. Mice in the placebo group were immunized with Montanide ISA 51/VSSP. The castrated group maintained a stable tumor inhibition until day 7 (60\%). However, at day 90 , all the mice exhibited a significant tumor re-growth and had to be sacrificed. The vaccinated group exhibited inhibition of tumor growth in 2 out of 5 animals (40\%) until day 100, which demonstrates a significant survival time, compared with the placebo and castrated mice $(\mathrm{P}<0.01)$. VSSP, very small size proteoliposomes; GnRHm1-TT, gonadotropin-releasing hormone mimetic peptide.

In order to determine the possible involvement of $\mathrm{T}$ cells in the immunological recognition of PABPN1, in addition to the analysis of anti-PABPN1 antibodies, the present study performed an anti-IFN- $\gamma$ ELISPOT using splenocytes collected from the sacrificed mice. Lymphocytes were extracted at the same time to act as controls. The results demonstrated a T-cell immune response directed to PABPN1 in mice that had developed anti-PABPN1 antibodies (data not shown).

\section{Discussion}

GnRH-based vaccines represent a promising anti-hormonal alternative treatment for patients with prostate cancer, since they reduce serum testosterone levels, leading to the generation of a memory immune response in vaccinated individuals $(6,10-12)$. However, the poor immunogenicity of small and autologous peptides, such as GnRH and its mimetics, is a challenging obstacle that must be overcome by immunologists $(10,11,24,25)$.

The combination of potent adjuvants to emulsify poor immunogenic antigens is an attractive approach that may promote an inflammatory cell environment, and is useful for the development of efficacious vaccines (26-28). A recent study demonstrated the usefulness of the GnRHm1-TT peptide adjuvanted with Montanide ISA 51 and VSSP to promote $100 \%$ immunogenicity and testosterone decrease in healthy adult animals in $<60$ days (12). However, the simulation of these effects in a tumoral mouse model that mimics prostate cancer represents an important challenge, which, to the best of our knowledge, has not been investigated thus far. 
A

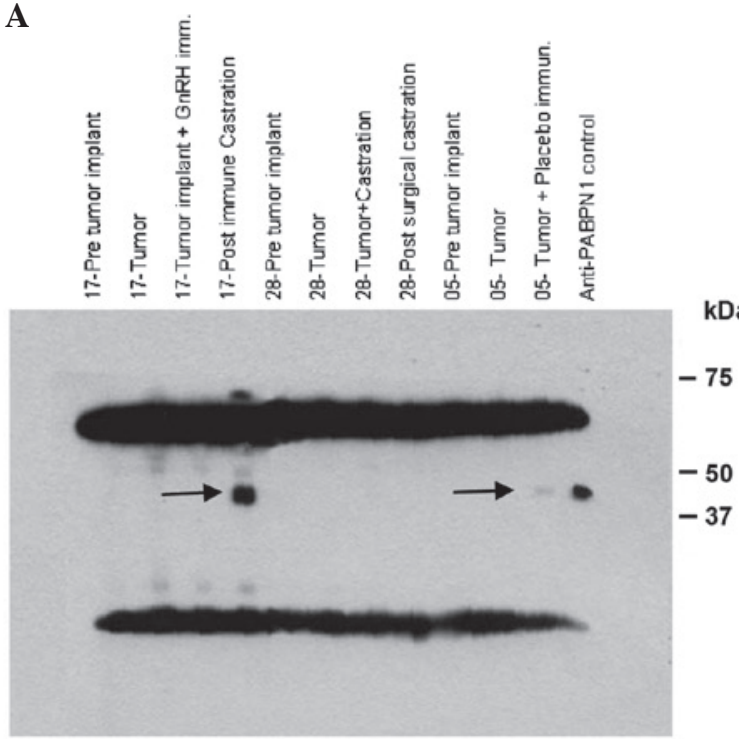

C
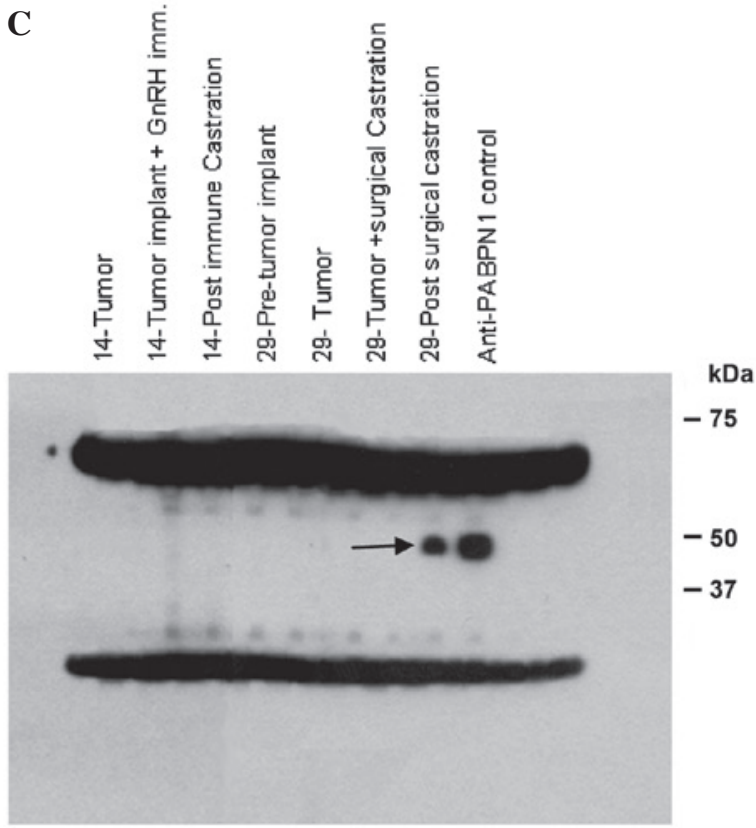

B

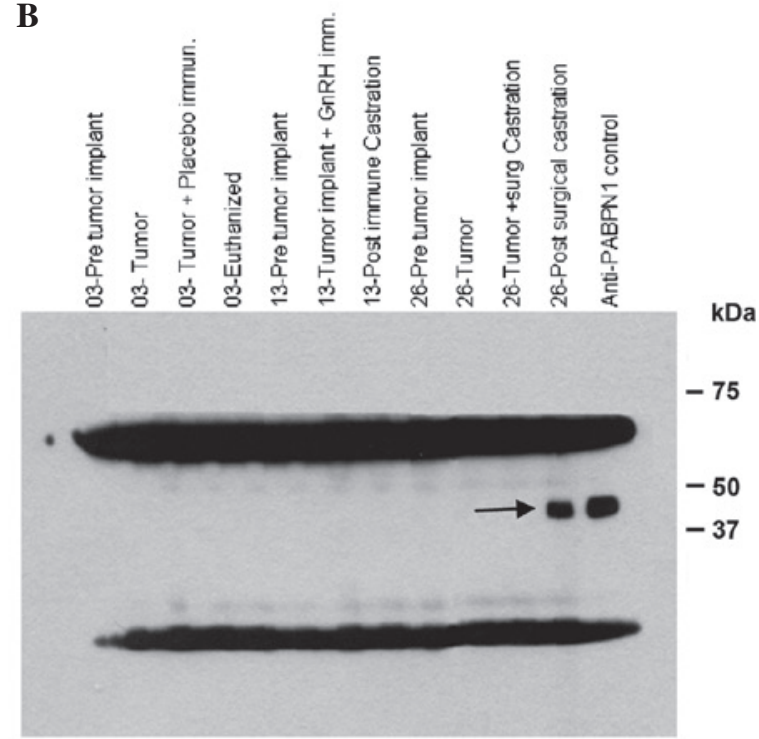

D
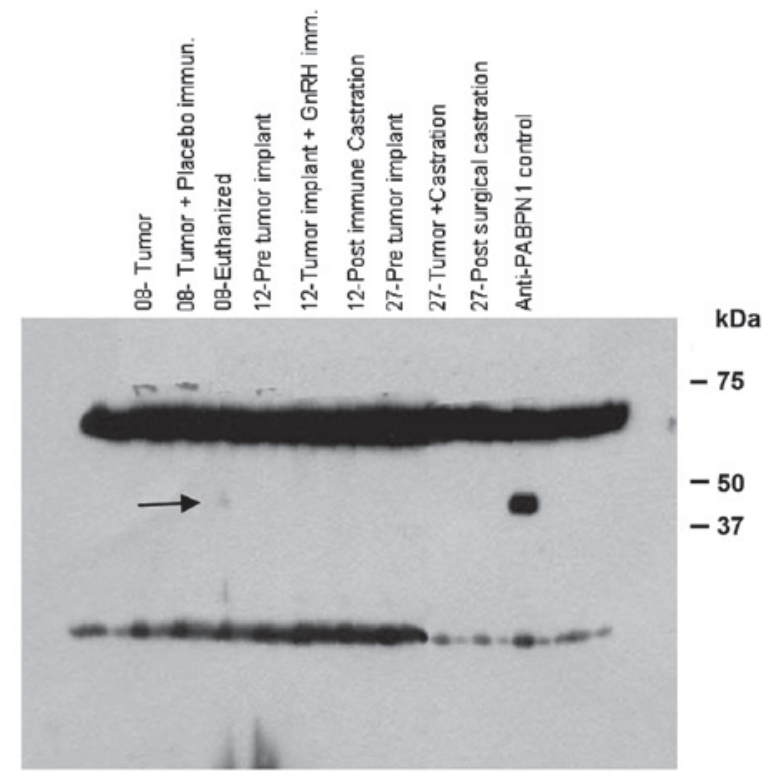

Figure 3. Western blot analysis of serum from mice injected with Shionogi carcinoma 115 cells, which was probed against Shionogi tumor lysate to determine recognition of the PABPN1 protein. Serum was collected from 5 mice from various treatment groups at serial time points, including pre-tumor implantation, tumor implantation, pre-castration, post-castration and sacrifice. Western blot analysis demonstrated autoantibody responses to a $44 \mathrm{kDa}$ protein derived from the murine Shionogi tumor model (shown by the black arrow) in mice numbers (A) 5, placebo and 17, immunized, (B) 26, surgical castration, (C) 29, surgical castration and (D) 8, placebo. Presence of autoantibodies following treatment was observed, which appeared to be associated with a poor prognosis Intense bands at the top and bottom of the gel represents staining of the heavy and light chains of antibodies contained in the tumors of the mice. GnRH, gonadotropin-releasing hormone; PABN1, polyadenylate-binding protein nuclear 1; immun., immunization; implant, implantation.

The murine SC 115 mouse model mimics the initial hormone-sensitive stage of prostate cancer and the development of CRPC in humans, and is a useful model to evaluate the effect of immunocastration (22). In order to ensure the Shionogi tumor implantation in the mice and produce an effective immune response to $\mathrm{GnRH}$, the present study performed three immunizations prior to tumor implantation, and three additional immunizations following implantation.

As previously demonstrated in healthy rats $(6,12)$, the DD/S mice implanted with the SC 115 tumor in the present study generated a rapid anti-GnRH seroconversion and high antibody titers following the third injection of SC 115 cells. This humoral immune response was associated with a significant decrease in testosterone levels. In 1 mouse the decrease in testosterone levels was similar to the decrease observed in the castration group. However, in the other 4 mice, the levels of testosterone were significantly reduced compared with the placebo mice, although not as reduced as in the castrated mice.

The effective immune response observed in tumor-implanted mice was similar to the immune response previously reported in healthy animals (12), and is possibly due to the use of a mixture of type 1 (Montanide ISA 51) and type 2 (VSSP) adjuvants. This observation is in agreement with previous studies describing the use of gram-negative bacteria-derived adjuvants and VSSP to 
promote cell antigen presentation in patients with cancer and immunodeficient patients $(24,25,29,30)$.

All the mice in the placebo group in the present study exhibited rapid tumor growth, and had to be sacrificed at 19-31 days. All the castrated animals exhibited a reduction in tumor volume, as expected in this hormone-sensitive prostate cancer model. In total, 3 of the mice immunized against GnRH also exhibited a reduction in tumor growth. However, 2 mice failed to respond, and exhibited tumor growth similar to that observed in the placebo group. Thus, they had to be sacrificed at days 30 and 35 . In contrast to the castrated animals, which all exhibited tumor recurrence, the 3 remaining mice in the GnRH immunized group exhibited no tumor recurrence. In total, 1 mouse had to be sacrificed at day 55 due to self-mutilation, but the other 2 mice had no regrowth up to day 100 , when the present study was terminated.

A previous study supports the role of testosterone ablation as a promoter of the immune response to prostate antigens and suggests that ADT stimulates quantitatively and qualitatively the immune system, and mitigates prostate antigen tolerance (23). To examine this hypothesis, the present study performed western blot and ELISPOT analyses to evaluate the humoral and cluster of differentiation $8^{+} \mathrm{T}$-cell immune response, respectively, in SC 115-implanted DD/S mice vaccinated with the GnRHm1-TT/Montanide ISA 51/VSS candidate.

Notably, PABPN1, a ubiquitously expressed protein that is involved in the polyadenylation of messenger RNA in eukaryotes, was observed to be present in the serum of SC 115-implanted DD/S mice subjected to various treatments. Mice with increased anti-PABPN1 antibody titers exhibited higher tumor growth rates. These findings may be associated with the tumor suppression mechanisms that operate in the host, which impede the immune response against tumor antigens (9). To associate these results with the cellular immune response, the present study investigated whether the animals that generated humoral anti-PABPN1 immune response also developed T-cell recognition of the PABPN1 protein. A T-cell immune response was revealed to be directed against the PABPN1 protein in mice that had developed anti-PABPN1 antibodies. The authors of the present study are currently investigating whether B- and T-cell responses to PABPN1 actively promote tumor recurrence in the murine SC 115 model, or are markers of other biological processes associated with tumor recurrence.

In conclusion, the present study has demonstrated that gradually lowering testosterone to moderate levels using immunization against $\mathrm{GnRH}$ appears to protect against prostate cancer recurrence, which is in agreement with the rapid and complete inhibition observed in castration and $\mathrm{GnRH}$ analogue therapy. More detailed and extensive studies are required to confirm this possibility of immune neutralization of GnRH as an improved treatment to reduce the rate of tumor recurrence in patients with androgen-insensitive prostate cancer.

\section{Acknowledgements}

The authors would like to thank the Union for International Cancer Control (Geneva, Switzerland; grant no., YY1/09/008/2009) for the fellowship received to support the present study. The authors would also like to thank the researchers at the Trev and Joyce
Deeley Research Centre (Victoria, Canada), British Columbia Cancer Research Centre (Vancouver, Canada) and University of Victoria (Victoria, Canada), particularly Professor Brad Nelson, Dr Julian Lum and Dr John Webb, for purchasing the mice and providing the laboratory facilities required to conduct the present study. The authors would also like to thank Mrs. Angela Lum and Mrs. Melanie Mawer at the Deeley Research Centre (Vancouver, Canada) for their contribution, and Mr. Orestes Padrón Yordi (Cuban Center for Genetic Engineering and Biotechnology, Havana, Cuba), Ms. Victoria Mazo Gray (University of Victoria; retired) and Mrs. Jean Howell (University of Victoria; retired) for correcting the manuscript. The present study was also supported by grants from the National Research Foundation (Pretoria, South Africa), University of Pretoria (Pretoria, South Africa) and University of Cape Town (Cape Town, South Africa).

\section{References}

1. Schröder FH: Prostate cancer around the world. An overview. Urol Oncol 28: 663-667, 2010.

2. Malvezzi M, Arfé A, Bertuccio P, Levi F, La Vecchia C and Negri E: European cancer mortality predictions for the year 2011. Ann Oncol 22: 947-956, 2011.

3. Pienta KJ and Bradley D: Mechanisms underlying the development of androgen-independent prostate cancer. Clin Cancer Res 12: 1665-1671, 2006.

4. Hirano D, Nagane Y, Satoh K, Mochida J, Sugimoto S, Ichinose T, Takahashi S, Maebayashi $\mathrm{T}$ and Saitoh T: Neoadjuvant LHRH analog plus estramustine phosphate combined with three-dimensional conformal radiotherapy for intermediate- to high-risk prostate cancer: A randomized study. Int Urol Nephrol 42: 81-88, 2010.

5. Labrie F: Keyrole of endocrinology in the victory against prostate cancer. Bull Cancer 93: 949-958, 2006 (In French).

6. Junco JA, Peschke P, Zuna I, Ehemann V, Fuentes F, Bover E, Pimentel E, Basulto R, Reyes O, Calzada L, et al: Immunotherapy of prostate cancer in a murine model using a novel $\mathrm{GnRH}$ based vaccine candidate. Vaccine 25: 8460-8468, 2007.

7. Miyake H, Nelson C, Rennie PS and Gleave ME: Testosterone-repressed prostate message- 2 is an antiapoptotic gene involved in progression to androgen independence in prostate cancer. Cancer Res 60: 170-176, 2000.

8. So AI, Bowden M and Gleave M: Effect of time of castration and tumour volume on time to androgen-independent recurrence in Shionogi tumours. BJU Int 93: 845-850, 2004.

9. Hahn S, Nesslinger NJ, Drapala RJ, Bowden M, Rennie PS, Pai HH, Ludgate C and Nelson BH: Castration induces autoantibody and $\mathrm{T}$ cell responses that correlate with inferior outcomes in an androgen-dependent murine tumor model. Int J Cancer 125: 2871-2878, 2009.

10. Talwar GP, Raina K, Gupta JC, Ray R, Wadhwa S and Ali MM: A recombinant luteinising-hormone-releasing-h ormone immunogen bioeffective in causing prostatic atrophy. Vaccine 22: 3713-3721, 2004.

11. Talwar GP, Vyas HK, Purswani $S$ and Gupta JC: Gonadotropin-releasing hormone/human chorionic gonadotropin beta based recombinant antibodies and vaccines. J Reprod Immunol 83: 158-163, 2009.

12. Aguilar FF, Barranco JJ, Fuentes EB, Aguilera LC, Sáez YL, Santana MD, Vázquez EP, Baker RB, Acosta OR, Pérez HG and Nieto GG: Very small size proteoliposomes (VSSP) and Montanide combination enhance the humoral immuno response in a GnRH based vaccine directed to prostate cancer. Vaccine 30: 6595-6599, 2012.

13. Ferro VA and Stimson WH: Investigation into suitable carrier molecules for use in an anti-gonadotrophin releasing hormone vaccine. Vaccine 16: 1095-1102, 1998.

14. Ko EC, Wang X and Ferrone S: Immunotherapy of malignant diseases. Challenges and strategies. Int Arch Allergy Immunol 132: 294-309, 2003.

15. Lage A, Perez R and Fernandez LE: Therapeutic cancer vaccines: At midway between immunology and pharmacology. Curr Cancer Drug Targets 5: 611-627, 2005. 
16. Clements CJ and Griffiths E: The global impact of vaccines containing aluminium adjuvants. Vaccine 20 (Suppl 3): S24-S33, 2002.

17. Lell B, Agnandji S, von Glasenapp I, Haertle S, Oyakhiromen S, Issifou S, Vekemans J, Leach A, Lievens M, Dubois MC, et al: A randomized trial assessing the safety and immunogenicity of AS01 and AS02 adjuvanted RTS,S malaria vaccine candidates in children in Gabon. PLoS One 4: e7611,2009.

18. Giannini SL, Hanon E, Moris P, Van Mechelen M, Morel S, Dessy F, Fourneau MA, Colau B, Suzich J, Losonksy G, et al: Enhanced humoral and memory B cellular immunity using HPV16/18 L1 VLP vaccine formulated with the MPL/aluminium salt combination (AS04) compared to aluminium salt only. Vaccine 24: 5937-5949, 2006.

19. Didierlaurent AM, Morel S, Lockman L, Giannini SL, Bisteau M, Carlsen H, Kielland A, Vosters O, Vanderheyde N, Schiavetti F, et al: AS04, an aluminum salt- and TLR4 agonist-based adjuvant system, induces a transient localized innate immune response leading to enhanced adaptive immunity. J Immunol 183: 6186-6197, 2009.

20. Schwarz TF: Clinical update of the AS04-adjuvanted human papillomavirus-16/18 cervical cancer vaccine, Cervarix. Adv Ther 26 983-998, 2009.

21. Rennie PS, Bruchovsky N, Buttyan R, Benson M and Cheng $\mathrm{H}$ : Gene expression during the early phases of regression of the androgen-dependent Shionogi mouse mammary carcinoma. Cancer Res 48: 6309-6312, 1988.

22. Nesslinger NJ, Sahota RA, Stone B, Johnson K, Chima N, King C, Rasmussen D, Bishop D, Rennie PS, Gleave M, et al: Standard treatments induce antigen-specific immune responses in prostate cancer. Clin Cancer Res 13: 1493-1502, 2007.

23. Nesslinger NJ, Ng A, Tsang KY, Ferrara T, Schlom J, Gulley JL and Nelson BH: A viral vaccine encoding prostate-specific antigen induces antigen spreading to a common set of self-proteins in prostate cancer patients. Clin Cancer Res 16: 4046-4056, 2010
24. Carr A, Mazorra Z, Alonso DF, Mesa C, Valiente O, Gomez DE, Perez R and Fernandez LE: A purified GM3 ganglioside conjugated vaccine induces specific, adjuvant-dependent and non-transient antitumour activity against B16 mouse melanoma in vitro and in vivo. Melanoma Res 11: 219-227, 2001.

25. Matzinger P: Tolerance, danger, and the extended family. Annu Rev Immunol 12: 991-1045, 1994.

26. Ambrosino DM, Bolon D, Collard H, Van Etten R, Kanchana MV and Finberg RW: Effect of Haemophilus influenzae polysaccharide outer membrane protein complex conjugate vaccine on macrophages. J Immunol 149: 3978-3983, 1992.

27. Udono $\mathrm{H}$ and Srivastava PK: Heat shock protein 70 -associated peptides elicit specific cancer immunity. J Exp Med 178: 1391-1396, 1993.

28. Nestle FO, Alijagic S, Gilliet M, Sun Y, Grabbe S, Dummer R, Burg G and Schadendorf D: Vaccination of melanoma patients with peptide- or tumor lysate-pulsed dendritic cells. Nat Med 4: 328-332, 1998.

29. Andersen BM: Endotoxin release from Neisseria meningitidis. Relationship between key bacterial characteristics and meningococcal disease. Scand J Infect Dis Suppl 64 (S64): 1-43, 1989.

30. Zollinger WD: New and improved vaccines against meningococal disease. In: New Generation Vaccines. Woodrow GC and Levine MM (eds). Marcel Dekker, Inc., New York, NY, pp325-348, 1990. 